\title{
DEPENDENCIES BETWEEN MODEL INDICATORS OF THE BASIC AND THE SPECIALIZED SPEED IN HOCKEY PLAYERS AGED 13-14
}

\author{
A. Antonov* \\ Department Football and Tennis, Sector Hockey, National Sports Academy “Vassil Levski” \\ Sofia, Bulgaria
}

\begin{abstract}
PURPOSE The aim of our research is to establish the degrees and directions of dependence between the main forms of manifestation of the basic and specialized speed. The subject was 20 boys born in 2005 and 2006.

METHODS Through tests and variance analysis (VA) we determine the level of the main forms of the basic and specialized speed, and through correlation analysis (CA) and intercriteria analysis (ICrA) we establish the existence of possible connections and degrees of dependence between them.

RESULTS: By using VA we were able to characterize the average level, the level of dispersion and the distribution of the values of the studied indicators.

Correlations and dependencies have been established by using CA, traditionally applied by sports science. We have also applied the innovative ICrA in order to confirm or reject the established correlations, directions and degrees of dependence.

CONCLUSIONS: The conducted CA and ICrA have shown that the means for the development of the basic linear speed (BLS) and specialized linear speed (SLS) do not have a significant impact on the development of the special complex speed. ICrA also indicates a lack of correlations and dependencies between BLS and SLS, which, however, is not confirmed by CA.
\end{abstract}

Key words: basic speed, Correlation analysis, InterCriteria Analysis, model indicators, hockey, Variance analysis

\section{INTRODUCTION}

The dynamics and intensification of game actions in modern hockey set high standards for the level of development of the basic speed (BS) - in running without a stick, the special speed - in running with a stick, and the specialized speed (SS) of players when dribbling and controlling the ball $(1,2)$. The speed of reaction and the speed of movement on the field are a huge advantage in a number of game situations - blocking a "drag flick" in defence in case of a penalty corner, successful performance of a quick attack, counterattack, effective press, securing or covering in defence, etc. (3).

\footnotetext{
*Correspondence to: Antonio Antonov, Department Football and Tennis, Sector Hockey, National Sports Academy "Vassil Levski", Sofia, Bulgaria, Email: antonio_hockey@yahoo.com, Mobile: + 359876666644
}

The speed of movement in nature, including in sport, is an indicator / expression of the speed of processes and phenomena and is characterized by acceleration $(a)$ - the rapid change in the speed of movement $\left({ }^{\vec{a}=}=\frac{\vec{v}-\overrightarrow{v_{0}}}{t}\right)$ in space and time (4)

In sports literature, science and practice there are many different definitions of the term speed. However, we can distinguish three main groups of definitions that most fully reflect the essence of speed, as follows:

- the ability of a person to perform motor actions for a minimum period of time (5);

- the ability of a person to react quickly to external stimuli and to execute fast start of the movements (6)

- a quality of a person, which is manifested in three main forms: 1 . the speed of a separate movement at low resistance; 2 . the frequency 


\section{ANTONOV A.}

of single movements at a small amplitude; 3 . the latent time of the motor reaction (7).

In our opinion, the last definition is the most comprehensive one and encompasses all forms of manifestation of speed, except for one - the rapid (abrupt) change of direction and pace of movement, i.e., sharpness. Sharpness is an especially important form of manifestation of speed in sports, also called by some authors: coordination speed, motor dexterity or coordination dexterity. The latter is logical due to the high correlation between speed, agility and coordination abilities, and perhaps sharpness is indeed the connection between speed and agility. With regard to players' speed abilities, a number of authors use the term coordination speed, which means the speed of movement of the body or of its parts in space when changing the structure of movements, i.e. the speedy execution of coordinated complex movements. $(8,9)$. According to the latter and other authors, the coordination speed is most often manifested in sports games in the rapidly changing conditions of the game or in combination with sports technique, which is accompanied by a change of direction, running zigzag, elimination movements, reliable control over the ball, etc. $(10,11)$

According to M. Gadev $(12,13)$, depending on the inert state of the body, at the maximum intensity of performance of speed abilities, the latter are subdivided into: starting acceleration and maximum speed when running in a straight line, in a curve or with a change of direction. The specificity of these speed abilities is expressed, first of all, in the differences of the constituent components of the forms of manifestation. These differences are an expression of the motor goals associated with the various inert states of the body. In the first form of manifestation, the goal is related to starting and accelerating the body and its parts with significant static inertia of the body, and the second form - it is related to developing and maintaining the maximum speed in case of significantly accelerated body and insignificant inertia.

According to V. Georgieva (14), specific manifestations of speed abilities in hockey are the starting speed and the special (coordination) speed. They characterize the manifestation of speed in the dynamically changing conditions of the game in the form of linear, starting or coordination speed, requiring frequent change of pace and direction of movement in combination with coordinationally complex technical and tactical skills. These types of speed are part of hockey players' speed abilities and have a significant place in their fitness status.

As a confirmation of what has been already pointed out, Hassan IHI (15) argues that starting acceleration and rapid change of direction are important speed qualities in all team sports. According to the same author, during a field game play hockey players run an average of 30 sprints (sections) with duration of 1.8 up to $4.1 \mathrm{~s}$, with a length of 10 to $30 \mathrm{~m}$ and frequency / intervals between the laps of $20 \mathrm{~s}$ up to $60 \mathrm{~s}$. The total distance run with maximum intensity at a "sprint speed" varies from $700 \mathrm{~m}$ to $1000 \mathrm{~m}$.

From what has been mentioned so far, we should be aware that speed is a complex quality that manifests itself through:

- $\quad$ latent time (speed) of motor reactions;

- frequency of movements;

- the speed of execution of single movements;

- $\quad$ rapid start and change of direction (sharpness).

In determining the priority forms for the development of speed, some specific features must be taken into account:

1. In which of the three main disciplines: indoor hockey, hockey 5 or hockey 11 , will we specialize when working for the development of speed?

2. The speed of hockey players always manifests itself as special or specialized. What are the correlations between the different forms of basic speed (BS) in running without a stick and a ball, of special speed (SpS) with a stick, and of specialized speed (SS) in speed dribbling?

The selection of forms, methods and means for the development of speed, should always take into account the fact that within competitive conditions, players change the direction of their movement every 5 seconds. In hockey 5 and indoor hockey, the intervals are probably shorter.

The variety of definitions, forms, means and methods for the development of speed are a 
prerequisite for seeking answers to questions such as:

1. How does carrying of the stick and dribbling the ball affect the running speed of the hockey player and in what degrees and directions of dependence do the different forms of manifestation of BS and SS enter?

2. What is the impact of the main indicators of BS on the hockey player's SpS and SS, i.e. the influence of the level of the starting acceleration, the maximum speed and the complex manifestation of the speed of movement without a ball on the speed movement with a stick and the speed dribbling?

3. Which form of manifestation of BS, SpS or $\mathrm{SS}$ : reaction speed, frequency of movements, starting acceleration, maximum speed, sharpness - the coordination speed or speed endurance is/are of priority?

The questions and the search for answers related to the dependences between the model indicators characterizing the non-specific and specific motor speed abilities of adolescent hockey players will always be relevant for science and practice.

\section{PURPOSE}

The purpose of our study is to determine the degrees and directions of dependence between the main forms of manifestation of the basic and specialized speed in hockey players aged 13-14 by applying CA and ICrA and comparing the results of the two analyses.

To achieve this purpose, we set the following tasks:

1. Determining the average values, the dispersion and the distribution of the basic indicators characterizing the level of BS and SS;

2. Determining significant correlations between the average values of the indicators we studied.

3. Determining significant intercriteria dependencies between the average values of the indicators we studied;

4. Comparing the results of CA and ICrA and detection of homogeneous (similar) and heterogeneous (dissimilar) dependencies.

The subject of the study were 20 boys -10 born in 2005 (aged 14 at the time of the study) and 10 born in 2006 (aged 13 at the time of the study), systematically playing hockey and included in the expanded composition of the national team in hockey for juniors U15 in the Youth Olympic discipline - Hockey 5.

The study focuses on the level of basic indicators characterizing the different forms of BS of hockey players - manifested in running without a stick, and of SB - manifested in speed dribbling.

\section{METHODS}

We established the level of BB and SB by conducting several tests. The tests presented in Table 1 give us objective information about the state of the main forms of manifestation of speed, namely: starting speed / acceleration (SS / SA) - test SR15, maximum speed (MS) test FS15, complex speed (CS) - test SR30, specialized starting speed / acceleration in dribbling (SSS / SSA) - test SFD15, specialized maximum speed in dribbling (SMS) - test FDFS15, complex specialized speed in dribbling (CSS) - test SFD30, specialized coordination speed (SCS) - LSC test ("Santi Cortes Labyrinth" - speed forehand dribbling with change of direction).

\section{Variance analysis (VA)}

The variance analysis used in the present study is based on common descriptive statistics analysis and aims to establish the average, dispersion and distribution of the indicator values (16) and to what extent the results of their distribution and dispersion are within the norm. The established results with regard to dispersion values enable us to evaluate them as homogeneous (coefficient of variance V\% $\leq$ 12), fairly homogenous (V\% 12-30) and strongly / highly inhomogeneous (V\% > 30).

\section{Correlation analysis (CA)}

Establishing the correlations and the degree of dependence between the main indicators of basic and specialized speed, has a significant role in the development of methodologies, work plans and programs for sports training of hockey players aged 13-14, emphasizing the development of speed and potential of adolescents. We have established correlations and dependencies with the help of an analysis that is traditionally applied in sports science, namely, correlation analysis (CA) by Pearson's coefficient (r) for ordinary in appearance, linear in shape and quantifiable features at the following degrees of correlation: weak ( $\mathrm{r}$ from 0 to 0.3 ), moderate ( $\mathrm{r}$ from 0.3 to 0.5 ), significant ( $\mathrm{r}$ from 0.5 to 0.7 ), strong ( $\mathrm{r}$ from 0.7 to 0.9 ) and very strong (r over 0.9 ). 


\section{InterCriteria Analysis}

As an innovative approach in our study we have applied InterCriteria analysis (ICrA), through which we will confirm or reject the established relationships, directions and degrees of dependence.

ICrA is a mathematical approach for finding dependencies (correlations) or the absence of such between pre-defined criteria (or parameters, or what is needed depending on the specific task), based on evaluations of objects according to these criteria. The approach is based on the theories of intuitionistic fuzzy logic and indexed matrices. The complete description of the approach has been given in (17). Table 2 presents the interpretation of the values of the degrees of agreement between criteria themselves, based on (18)

Table 1. Tests for control and evaluation of basic and specialized speed in hockey

\begin{tabular}{llccc}
\hline № & Name and abbreviation of the test & Unit & Accuracy & Direction \\
\hline 1 & Sprint running $-15 \mathrm{~m}$ (SR15) & $\mathrm{s}$ & 0.01 & - \\
\hline 2 & Sprint running $-30 \mathrm{~m}$ (SR30) & $\mathrm{s}$ & 0.01 & - \\
\hline 3 & Flying start $-15 \mathrm{~m}$ (FS15) & $\mathrm{s}$ & 0.01 & - \\
\hline 4 & Sprint forehand dribbling $-15 \mathrm{~m}$ (SFD15) & $\mathrm{s}$ & 0.01 & - \\
\hline 5 & Sprint forehand dribbling $-30 \mathrm{~m}$ (SFD30) & $\mathrm{s}$ & 0.01 & - \\
\hline 6 & Forehand dribbling flying start $-15 \mathrm{~m}$ (FDFS15) & $\mathrm{s}$ & 0.01 & - \\
\hline 7 & Labyrinth of Santi Cortes (LSC) & $\mathrm{s}$ & 0.01 & - \\
\hline
\end{tabular}

Table 2. Interpretation of the values of degree of "agreement" (ICrA consonance and dissonance scale)

\begin{tabular}{|c|c|}
\hline$\mu_{C_{k}, C_{l}}$ & Meaning \\
\hline$[0.00-0.05]$ & strong negative consonance, (SNC) \\
\hline$(0.05-0.15]$ & negative consonance, $(\mathrm{NC})$ \\
\hline$(0.15-0.25]$ & weak negative consonance, (WNC) \\
\hline$(0.25-0.33]$ & weak dissonance, (WD) \\
\hline$(0.33-0.43]$ & dissonance, $(\mathrm{D})$ \\
\hline$(0.43-0.57]$ & strong dissonance, $(\mathrm{SD})$ \\
\hline$(0.57-0.67]$ & dissonance, $(\mathrm{D})$ \\
\hline$(0.67-0.75]$ & weak dissonance, (WD) \\
\hline$(0.75-0.85]$ & weak positive consonance, (WPC) \\
\hline$(0.85-0.95]$ & positive consonance, $(\mathrm{PC})$ \\
\hline$(0.95-1.00]$ & strong positive consonance, (SPC) \\
\hline
\end{tabular}

When interpreting the results, the values of $v_{C_{1}, C_{1}}$, respectively $\pi_{C_{k}, C_{l}}$ should be considered.

The ICrA can be applied to decision making in different areas of knowledge, e.g. in medicine (19), mathematics (20), sport (2123), etc.

The establishment of the relations, similarity of behaviour and degrees of dependence has been carried out in the following directions:

1. Determining significant, strong and very strong correlations between the examined indicators characterizing the different forms of manifestation of the basic and specialized speed as well as searching for similar relationships in both age groups;

2. InterCtiteria analysis of negative and positive consonance, i.e. weak and strong, between basic speed and specialized speed indicators of hockey players aged 13-14.

\section{RESULTS}

Tables 3 and 4 present the statistical data on the average level and the dispersion of the values of the indicators characterizing the level of the forms of manifestation of the basic and specialized speed we studied, separately for players aged 13 born in 2006 (Table 3) and for players aged 14 born in 2005 (Table 4). 
ANTONOV A.

Table 3 Variation values of the indicators of basic and specialized speed of players aged 13

\begin{tabular}{lccccc}
\hline TESTS/INDICATORS (U13) & $\mathrm{X}(\mathrm{s})$ & $\mathrm{S}$ & $\mathrm{R}$ & $\mathrm{V} \%$ & $\mathrm{Ex}$ \\
\hline SR15/ SS & 3.02 & 0.30 & 1.03 & 9.91 & 1.01 \\
\hline FS15/ MS & 2.38 & 0.26 & 0.82 & 11.07 & -0.97 \\
\hline SR30/ CS & 5.40 & 0.46 & 1.21 & 8.59 & -1.08 \\
\hline SFD15/ SSS & 3.52 & 0.41 & 1.44 & 11.57 & 0.77 \\
\hline FDFS15/ SMS & 2.90 & 0.32 & 0.98 & 10.87 & -0.96 \\
\hline SFD30/ CSS & 6.42 & 0.67 & 2.30 & 10.44 & -0.19 \\
\hline LSC/ SCS & 22.89 & 2.13 & 6.59 & 9.29 & 0.19 \\
\hline
\end{tabular}

Table 4 Variation values of the indicators of basic and specialized speed of players aged 14

\begin{tabular}{llllll}
\hline TESTS/INDICATORS (U13) & $\mathrm{X}(\mathrm{s})$ & $\mathrm{S}$ & $\mathrm{R}$ & $\mathrm{V} \%$ & $\mathrm{Ex}$ \\
\hline SR15/ SS & 2.87 & 0.22 & 0.62 & 7.83 & -1.09 \\
\hline FS15/ MS & 2.16 & 0.25 & 0.82 & 11.49 & 1.02 \\
\hline SR30/ CS & 5.03 & 0.41 & 1.32 & 8.13 & 0.58 \\
\hline SFD15/ SSS & 3.40 & 0.24 & 0.76 & 7.13 & -0.43 \\
\hline FDFS15/ SMS & 2.52 & 0.19 & 0.52 & 7.65 & -1.03 \\
\hline SFD30/ CSS & 5.92 & 0.40 & 1.12 & 6.69 & -1.02 \\
\hline LSC/ SCS & 23.43 & 3.97 & 11.87 & 16.95 & -0.77 \\
\hline
\end{tabular}

Analysis of the values of the dispersion indicators: range $(\mathrm{R})$, standard deviation $(\mathrm{S})$ and coefficient of variation (V\%), provide us with a quantitative characteristics of the values variability.

The excess coefficient (Es) determines the distribution of the values of the investigated indicators in the vertical plane - their elevation or flatness with respect to the normal curve.

Tables 5 and 6 present in the form of correlation matrices the Pearson correlation coefficient (r). The established degrees of dependencies between the indicators are outlined as follows:

- $\quad$ no correlation ( $\mathrm{r}$ from 0.00 to 0.29 ) highlighted in pale green;

- insignificant / moderate correlation ( $\mathrm{r}$ from 0.30 to 0.49 ) highlighted in green;

- significant correlation ( $\mathrm{r}$ from 0.50 to 0.69 ) highlighted in yellow;

- $\quad$ strong correlation ( $\mathrm{r}$ from 0.70 to 0.89 ) - highlighted in orange;

- $\quad$ very strong correlation ( $\mathrm{r}$ over 0.9 ) highlighted in red.

Table 5. Correlation dependencies between the indicators of hockey players aged 13 born in 2006

\begin{tabular}{llllllll}
\hline TESTS/INDICATORS & SR15 & FS15 & SR30 & SFD15 & FDFS15 & SFD30 & LSC \\
\hline SR15/ SS & 1.00 & & & & & & \\
\hline FS15/ MS & 0.35 & 1.00 & & & & & \\
\hline SR30/ CS & 0.85 & 0.80 & 1.00 & & & & \\
\hline SFD15/ SSS & 0.39 & 0.88 & 0.75 & 1.00 & & & \\
\hline FDFS15/ SMS & 0.05 & 0.87 & 0.53 & 0.72 & 1.00 & & \\
\hline SFD30/ CSS & 0.26 & 0.94 & 0.70 & 0.94 & 0.91 & 1.00 & \\
\hline LSC/ SCS & 0.11 & -0.04 & 0.04 & -0.09 & 0.07 & -0.02 & 1.00 \\
\hline
\end{tabular}

Table 6. Correlation dependencies between the indicators of hockey players aged 14 born in 2005

\begin{tabular}{llllllll}
\hline TESTS/INDICATORS & SR15 & FS15 & SR30 & SFD15 & FDFS15 & SFD30 & LSC \\
\hline SR15/ SS & 1.00 & & & & & & \\
\hline FS15/ MS & 0.49 & 1.00 & & & & & \\
\hline SR30/ CS & 0.85 & 0.88 & 1.00 & & & & \\
\hline SFD15/ SSS & 0.70 & 0.82 & 0.88 & 1.00 & & & \\
\hline FDFS15/ SMS & 0.59 & 0.61 & 0.70 & 0.65 & 1.00 & & \\
\hline SFD30/ CSS & 0.72 & 0.80 & 0.88 & 0.93 & 0.89 & 1.00 & \\
\hline LSC/ SCS & 0.09 & 0.44 & 0.32 & 0.31 & 0.13 & 0.25 & 1.00 \\
\hline
\end{tabular}


The data obtained for players aged 13 (born 2006) has been subjected to ICrA, using the ICrAData software (24). The observed $\mu$ values (degree of agreement) are presented in Table 7 and are indicated as follows:

- $\quad$ dissonance, weak dissonance or strong dissonance (D, WD or SD) highlighted in green;
- weak positive or weak negative consonance (WPC or WNC) highlighted in yellow;

- $\quad$ positive or negative consonance, (PC or NC) highlighted in orange;

- $\quad$ strong positive or strong negative consonance, (SPC or SNC) highlighted in red.

Table 7. InterCriteria Analysis - dependencies between the indicators of players aged 13 (born 2006)

\begin{tabular}{llllllll}
\hline$\mu$-values & SR15 & FS15 & SR30 & SFD15 & FDFS15 & SFD30 & LSC \\
\hline SR15/ SS & 1.00 & & & & & & \\
\hline FS15/ MS & 0.67 & 1.00 & & & & & \\
\hline SR30/ CS & 0.84 & 0.82 & 1.00 & & & & \\
\hline SFD15/ SSS & 0.80 & 0.78 & 0.87 & 1.00 & & & \\
\hline FDFS15/ SMS & 0.64 & 0.62 & 0.71 & 0.73 & 1.00 & & \\
\hline SFD30/ CSS & 0.80 & 0.76 & 0.84 & 0.89 & 0.84 & 1.00 & \\
\hline LSC/ SCS & 0.53 & 0.60 & 0.60 & 0.60 & 0.60 & 0.58 & 1.00 \\
\hline
\end{tabular}

No significant degree of uncertainty $(\pi$ values) has been observed, so the results are reliable. The results obtained by ICrA show that correlation can be observed between the following indicators:

- $\quad$ positive consonance:

- SFD30 and SFD15 (highly correlated indicators);

- SFD15 and SR30

- $\quad$ weak positive consonance:

- SFD30 and SR30;

- SR30 and SR15;

- SFD30 and FDFS15;

- SR30 and FS;

- SR15 and SFD15;

- SR15 and SFD30;

$\circ \quad$ SFD15 and FS;

$\circ \quad$ SFD30 and FS (the least correlated).
The indicators in the remaining pairs are not related.

The same analysis has been performed over the data obtained for players aged 14 (born 2005). The observed $\mu$-values are presented in Table 8. In this case the following indicators correlations can be observed:

- $\quad$ positive consonance:

- SFD30 and FS (highly correlated indicators);

O SR30 and SR15;

SFD15 and FS;

- SFD30 and FDFS15;

- weak positive consonance:

$\circ \quad$ SFD30 and SFD15, FDFS15 and FS;

$\circ \quad$ SFD15 and SR30;

- SR30 and FS (the least correlated).

The indicators in the remaining pairs are not related.

Table 8. InterCriteria Analysis - dependencies between the indicators of players aged 14 (born 2005)

\begin{tabular}{cccccccc}
\hline$\mu$-values & SR15 & FS15 & SR30 & SFD15 & FDF15 & SFD30 & LSC \\
\hline SR15 & 1.00 & & & & & & \\
\hline FS15 & 0.64 & 1.00 & & & & & \\
\hline SR30 & 0.89 & 0.76 & 1.00 & & & & \\
\hline SFD15 & 0.67 & 0.87 & 0.78 & 1.00 & & & \\
\hline FDFS15 & 0.49 & 0.80 & 0.6 & 0.67 & 1.00 & & \\
\hline SFD30 & 0.58 & 0.93 & 0.69 & 0.80 & 0.87 & 1.00 & \\
\hline LSC & 0.56 & 0.49 & 0.56 & 0.44 & 0.49 & 0.47 & 1.00 \\
\hline
\end{tabular}




\section{DISCUSSION}

Speed in sports is a psychophysiological concept characterizing the degree of development of the already mentioned forms of manifestation of this quality: in non-specific conditions - as basic speed and in specific conditions - as special and specialized speed. It should be noted that in physiological aspect, the concept of "speed", as a characteristic of the potential of the individual, has been scientifically specified and defined quite thoroughly. At the same time, from a sportspedagogical point of view, it contains ambiguities, because as a scientific concept it is lacking content that is important in practice. In this sense, considering the speed and its specific and non-specific forms of manifestation, it is always necessary to keep in mind that on the one hand, each individual has its own genetic controllable growth of the socalled elementary forms of manifestation of speed, and on the other hand, that between the individual elementary forms of manifestation and in specific and non-specific conditions there is not always a reliable relationship.

\section{Characteristics - average level, dispersion and distribution of the indicators}

The variance analysis reflected in Tables 3 and 4 has allowed us to make a quantitative description of the state of the studied indicators by determining the average level, the level of dispersion and the distribution of the values of the studied indicators.

The analysis of the average values $(\mathrm{X})$ gives us a logical picture. In the players aged 14 the average values of the first 6 indicators, characterizing the different forms of the basic linear speed (BLS) and the specialized linear speed (SLS) are higher than those in the players aged 13. The average value of SCS in the 13 -year-olds $-X=22.89 \mathrm{~s}$ is higher than that of that in the 14-year-olds $-X=23.43 \mathrm{~s}$ (see Tables 3 and 4). Thus, we can assume that BS and SLS are probably not in high correlation with SCS, i.e. keeping the maximum speed with a sharp change of direction. It is very likely that dribbling technique and speed endurance had a significant impact on the level of SCS, established with the LSC test.

The range $(\mathrm{R})$, the standard deviation $(\mathrm{S})$ and the coefficient of variance $(\mathrm{V})$ give us reliable information about the dispersion of the homogeneity of the indicators. It can be seen that the highest value of the range $(\mathrm{R})$ is logically observed in the indicator with the highest arithmetic mean $-\mathrm{SCS}(\mathrm{R}-11.87 \mathrm{~s}$ in the 14-year-olds, $\mathrm{R}-6.59 \mathrm{~s}$ in the 13-yearolds), and the lowest - in the 15-meter sections, characterizing MS and SMS - FS15 $(\mathrm{R}-0.82 \mathrm{~s}$ in the 13-year-olds) and FDFS15 ( $\mathrm{R}-0.52 \mathrm{~s}$ in the 14-year-olds).

The coefficient of variance (V\%) expressing the dispersion of the feature in percentages and the homogeneity of the sample allows us to establish the following:

- In players aged 13, the dispersion of the values of all the studied indicators characterizing BS and SS is homogeneous;

- In players aged 14, the picture in the first 6 tests is identical. In SCS, the dispersion of the values is approximately uniform.

- In players aged 14, the values of SCS show the highest arithmetic mean $\mathrm{X}-23.43 \mathrm{sec}$, the highest value of the range $\mathrm{R}-11.87 \mathrm{~s}$ and the highest percentage of variation $\mathrm{V} \%-16.95$.

\section{Analysis of CA results - presence and absence of dependencies}

Table 5 and Figure 1 and 2 make it evident that in players aged 13, there is 1 pair (SS30 FDFS15) with significant correlation, 7 pairs (SR15 - SR30, FS15 - SR30, FS15 - SFD15, FS15 - FDFS15, SR30 - SFD15, SR30 SFD30, SFD15 - FDFS15) with strong correlation, 3 pairs (FS15 - SFD30, SFD15 SFD30, FDFS15 - SFD30) with very strong correlation, and in the other 10 pairs no significant correlations are observed. In sports science it is known that there are strong correlations between some forms of manifestation of speed, which has been confirmed in our scientific research as well. The following findings are of interest for sports science and practice:

- $\quad$ There is a lack of significant and stronger correlations between the indicator of SS (SR15) and MS (FS15), SSS (SDF15), SMS (FDFS15), CSS (SDF30), as well as with the indicator for SCS (LSC). The level of MS and SSS, SMS and SCS is lower than the required level for 13-year-olds. This hypothesis of ours has been confirmed by the very strong correlations between FS 15 and SS 30 , and the indicators of specialized speed. In our opinion, the low level of MS and the dribbling technique leads to a low level of all forms of SLS.

- $\quad$ The lack of any relationship between SCS (LSC) and all other speed indicators, confirms the hypothesis of the variation 


\section{ANTONOV A.}

analysis. It is evident that SCS does not correlate significantly with the studied forms of BLS and SLS - characterizing the speed of dribbling in a straight line.

Table 6 and Figure 1 and 2 make it evident that in players aged 14, there are 3 pairs (SR15 FDFS15, FS15 - FDFS15 and SFD15 FDFS15) with significant correlation, 10 pairs (SR15 - SR30, SR15 - SFD15, SR15 SFD30, FS15 - SR30, FS15 - SFD15, FS15 SFD30, SR30 - SFD15, SR30 - FDFS15, SR30 - SFD30 and FDFS15 - SFD30) with strong correlation, 1 pair (SFD15 - SFD30) with very strong correlation, and 7 pairs with no correlation between the indicators,

The following findings are of interest for sports science and practice:

- In all indicators characterizing BLS and SLS, there are significant, strong and very strong correlations, except between SA and MS (SB15 - FS15). This fact gives us reason to state that in order to develop SLS we can work with general development and special preparatory tools.

- There is a lack of significant correlation between the indicator of SA (SR15) and of MS (FS15), but the presence of correlations with the indicators of the specialized speed - SSA (SFD15), SMS (FDFS15), CSS (SFD30) shows a tendency for some improvement of the dribbling speed in the starting acceleration and in dribbling at maximum speed. However, it should be noted that the development of MS is not at the required level for 14-year-olds.

- There is a slight increase in the correlation between the indicator characterizing SCS - LSC and all the others, established by three moderate dependencies (LSC - FS, LSC - SR30 and LSC - SFD15). The lack of significant correlations between SCS and the other indicators of the 14-yearolds clearly shows that for development it is necessary to work with means close to competitive conditions, performing at maximum speed - dribbling with a rapid change of direction at intervals of 2 to 5 seconds, with a length of laps from $5 \mathrm{~m}$ to 30 $\mathrm{m}$ and a total duration of up to 20 seconds, as mentioned by Hassan IHI, 2018 in his research.

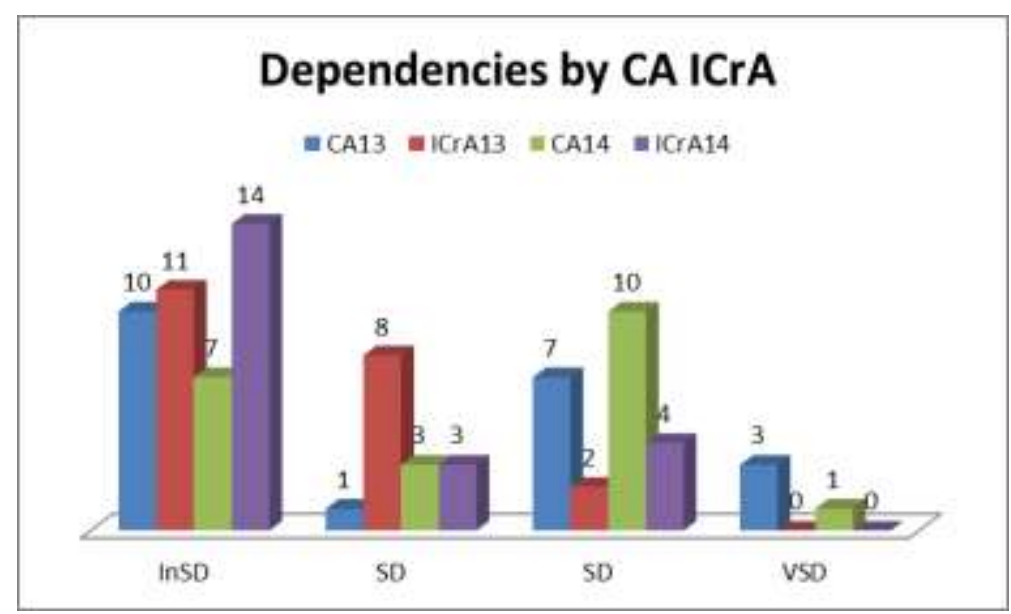

Figure 1 . Degrees of dependences between the indicators

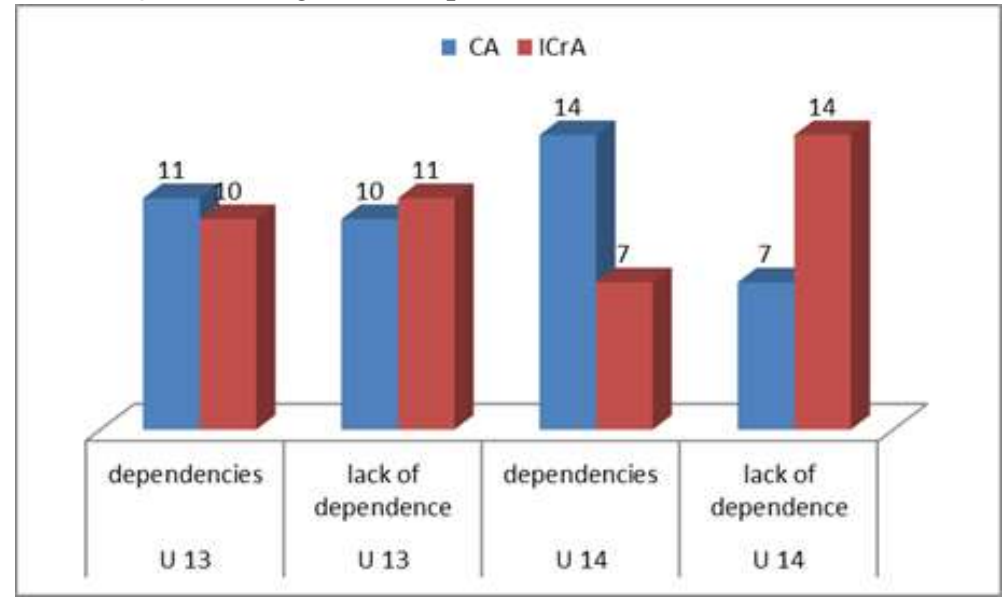

Figure 2. Detected dependencies on CA-ICrA 


\section{ICrA results}

The analysis of the results of the ICrA has shown the following results that are of interest for theory and practice:

- In the 13-year-olds there logically is a strong dependence between the indicators SCS (SFD30) and SSS (SFD15), which decreases to significant in the 14-year-olds;

- In the 13-year-olds there are nine logically significant dependences: SR30 and SR15, SR30 and FS15, SFD15 and SR15, SFD15 and FS15, SFD15 and SR30, SFD30 and SR15, SFD30 and FS15, SFD30 and SR30, SFD30 and FDFS15. In the 14-yearolds, four of them: SR30 and SR15, SFD15 and FS15, SFD30 and FS15, SFD30 and FDFS15 become strong, one - SFD 15 and SR 30 - retains its significant strength, and in the other four - SR30 and FS15, SFD15 and SR15,
SFD 30 and SR15, SFD30 and SR30 - the dependence is lost;

- $\quad$ The four very strong dependencies established by the CA are determined as significant and strong by the ICrA.

- The ICrA establishes a lack of dependence between some indicators of the BS and SS. An exception in 13-year-olds is the CSS, which correlates with all five indicators, while the CA had established dependences between four indicators. In the 14-year-olds, the CSS correlates with only three indicators: MS, SSS and SMS, whereas the CA had established dependence with all five;

- $\quad$ The ICrA confirms the lack of any relationship between SCS and other indicators, as established by the CA, both for 13- and 14year-olds.

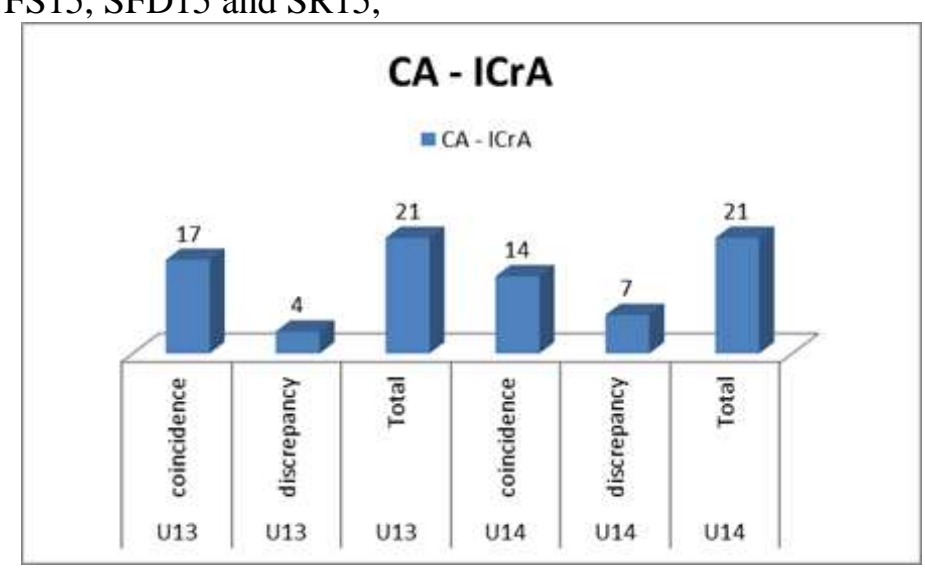

Figure 3. Coincidences and discrepancies

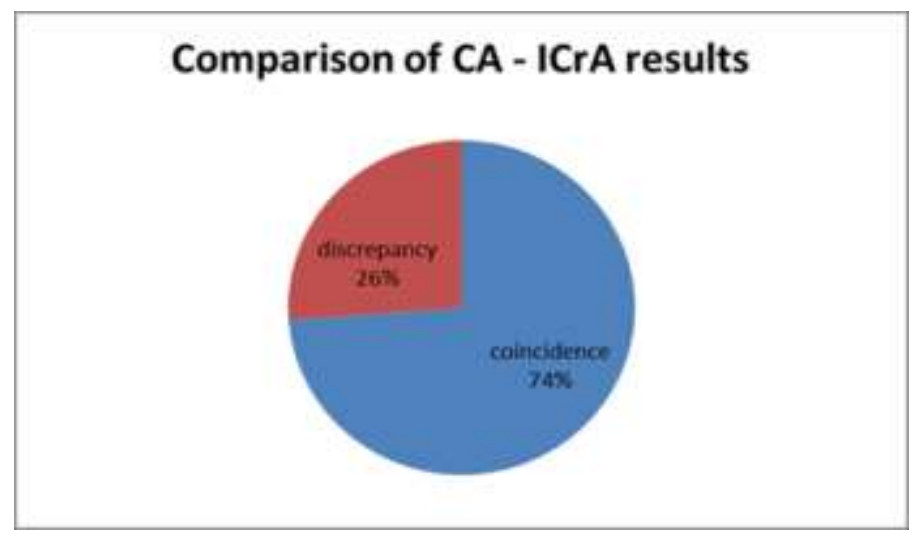

Figure 4. Comparison of $\mathrm{CA}$ and $\mathrm{ICrA}$ results

\section{CONCLUSION}

Both statistical methods - CA and ICrA prove that the means for the development of BLS and SLS will not have a significant impact on the development of SCS, characterized by dribbling/running with the ball at maximum speed with a rapid and frequent change of direction.
The differences in the correlations between some BLS and SLS indicators in the two age groups established by both analyzes show that in hockey players aged 13-14 the processes of development of the different forms of speed are uneven.

According to the CA, the presence of significant and strong dependencies between the three forms of basic and the three forms of 
specialized linear speed is an indicator that the general development and the special preparatory means for improving the speed without the ball will have an impact on the speed of dribbling in a straight line. The latter finding is not fully confirmed by ICrA.

The comparison of the results of CA and IcrA, as presented in Figures 3 and 4, shows that in $31(74 \%)$ out of 42 cases (17 among those born in 2005 and 14 among those born in 2006), the established dependencies overlap. In 11 cases $(26 \%)$ there is a significant discrepancy between the dependencies found by the two analyzes (presence or absence of dependence). The determined differences are mainly observed between some indicators of the basic and specialized speed. According to ICrA, the development of the linear running speed does not significantly affect the development of the linear running speed of dribbling, i.e., a technique has a stronger effect on dribbling/running with the ball than the basic speed at this age. In these cases, CA establishes significant or strong dependencies between BLS and SLS indicators.

To improve one of the most important forms of specialized speed, namely - SCS, we recommend working with game exercises close to competitive hockey game conditions. Exercises for the development of speedy dribbling technique must be performed at maximum speed, combined with a rapid change of direction at intervals of 2 to $5 \mathrm{~s}$, with a length of the laps from $5 \mathrm{~m}$ to $30 \mathrm{~m}$ and a total duration from 5 to $15 \mathrm{~s}$.

\section{ACKNOWLEDGEMENTS}

This presentation has been partially supported by the Bulgarian National Scientific Fund under Grant KP-06-N22/1 "Theoretical Research and Applications of InterCriteria Analysis".

\section{REFERENCES}

1. Antonov, A., Gadev, M., Dimitrov, L., Influence of the hockey stick on the manifestation of speed in 12-year-old field hockey players. NM spisanie "Sport $i$ Nauka”, 6:42-465, 2001 (in Bulgarian)

2. Tsarukha, V., Antonov, A., Development of physical qualities when working with 8-9 year old hockey player. Eighth International Scientific Conference at the Department of "Football and Tennis", pp. 44-47, Avangard Prima Sofia, 2011. (in Bulgarian)
3. Penny, L., Improve your hockey speed with this acceleration exercises. Planet Hockey Magazine, New Zealand, 2018, https://hockeyperformanceacademy.com/in crease-speed-in-field-hockey/

4. Zatsiorsky, V., Physical qualities of sportsmen. FS, M., 1970 (in Russian)

5. Stepanenkova, E.Y., Theory and methodology of children's physical education and development, 2001 (in Russian)

6. Popov, V.B., Special exercises in training athletes, 2003 (in Russian)

7. Zhelyazkov, T. S., Dasheva, D., Basics of Sports Training. BOLID-INS, S., 2017 (in Bulgarian)

8. Bachvarov, M., Sportology, Sofia, NSA, 2000 (in Bulgarian)

9. Brechue, W., Structure-function relationship performance and running speed in sport. International Sciences, 23(2):313350, 2011

10.Gadev, M., Nature and forms of manifestation of motor speeding potential in athletics. Journal „Leka atletika $i$ nauka", 1(13):134-139, 2013 (in Bulgarian)

11.Gadev, M., Peev, P., Tsvetkov, S., Chalakov, M., Ivanova, G., Monitoring of motor mobility in game environment in 1314 year old football players. S., NSA PRES, pp. 110, 2018 (in Bulgarian)

12.Gadev, M., Additional arguments on the category of speed. Journal ,Sport i nauka", 4-5:105-108, 1999 (in Bulgarian)

13.Gadev, M., Synergetic-conceptual model of conditioning training in football. S., BOLID-INS, p. 119, 2015 (in Bulgarian)

14.Georgieva, V., Diagnostics and assessment of the endurance rate of 14-16 year old hockey players. PhD thesis, SWU, 2018 (in Bulgarian)

15.Hassan, I.H.I., Relationship between strength, speed and change direction performance in field hockey players. $M O J$ Sports Med, 2(1):54-58, DOI: 10.15406/mojsm.2018.02.00046, 2018, https://medcraveonline.com/MOJSM/MOJS M-02-00046.pdf

16.Gigova, V., Shandurkova, S., Statistical methods in sports. Instructions for using the textbook, 2014 (in Bulgarian)

17.Atanassov, K., Mavrov, D., Atanassova, V. (2014) Intercriteria decision making: A new approach for multicriteria decision making, based on index matrices and intuitionistic fuzzy sets. Issues on Intuitionistic Fuzzy Sets and Generalized Nets, 11:1-8, 2014. 
18. Atanassov, K., Atanassova, V., Gluhchev, G., InterCriteria Analysis: ideas and problems. Notes on Intuitionistic Fuzzy Sets, 21(1):81-88, 2015.

19.Todinova, S., Mavrov, D., Krumova, S., Marinov, P., Atanassova, V., Atanassov, K., Taneva, S.G., Blood plasma thermograms dataset analysis by means of InterCriteria and correlation analyses for the case of colorectal cancer. International Journal Bioautomation, 20(1):115-124, 2016.

20.Roeva, O., Zoteva, D., Knowledge discovery from data: InterCriteria Analysis of mutation rate influence. Notes on Intuitionistic Fuzzy Sets, 24(1):120-130, 2018.

21.Antonov, A., Analysis and Detection of the Degrees and Direction of Correlations between Key Indicators of Physical Fitness of 10-12-year-old Hockey Players.
ANTONOV A.

International Journal Bioautomation, 23(3):303-314, 2019.

22. Antonov, A., Influence of the Indoor Hockey "Push \& Flick" methodology on the ball speed during the penalty corner shooting, Uncertainty and Imprecision in Decision Making and Decision Support: New Challenges, Solutions and Perspectives. Advances in Intelligent Systems and Computing, in press, 2020.

23.Antonov, A., Zoteva, D., Roeva, O., Influence of the "Push \& Flick" Methodology on the Accuracy of the Indoor Hockey Penalty Corner Shooting. Journal of Applied Sports Sciences, Vol. 1, in press, 2020.

24.Ikonomov, N., Vassilev, P., Roeva, O., ICrAData - Software for InterCriteria analysis. International Journal Bioautomation, 22(1):1-10, 2018. 